\title{
COPD
}

\section{Auch Patienten mit geringem Risiko profitieren von Tiotropium}

- Nach dem neuen GOLD-Update 2011 wird COPD heute nicht mehr nur anhand der Einschränkung der Lungenfunktion, sondern nach der Schwere der Symptome und dem Exazerbationsrisiko klassifiziert und therapiert. Lang wirksame Anticholinergika (LAMA) gelten für eine Dauertherapie als Medikamente der ersten Wahl.

Im neuen GOLD-Report wird die Behandlung mit LAMAs für die Patientengruppen B-D empfohlen, d.h. für Patienten, die aufgrund starker Symptome und/oder eines hohen Exazerbationsrisikos (abgeleitet aus der Häufigkeit vorangegangener Exazerbationen) eine Dauertherapie benötigen. Für Gruppe $A$, Patienten mit wenig Symptomen und geringem Exazerbationsrisiko, gelten LAMAs als zweite Option. Diese Empfehlung basiert ausschließlich auf Daten für Tiotropium $\left(\right.$ Spiriva $\left.^{\circledR}\right)$, erläuterte Prof. Antonio Anzueto,
San Antonio/USA. Tiotropium ist das bislang einzige in Deutschland für die COPDTherapie zugelassene LAMA und hat seine Effektivität und Sicherheit mit einer Gesamtbehandlungsdauer von über 25 Millionen Patientenjahren (Handihaler ${ }^{\circledR}$ und Respimat $^{\oplus}$ ) eindrucksvoll belegt.

\section{Management von Exazerbationen von zentraler Bedeutung}

Wie Prof. Claus Vogelmeier, Marburg, betonte, legt der aktualisierte GOLD-Bericht einen neuen Schwerpunkt auf das Management von Exazerbationen. Tiotropium ist in der Lage, bei Patienten aller GOLDKlassifikationen das Exazerbationsrisiko zu senken. Dies sei neben der Symptomkontrolle das wichtigste Therapieziel in der Behandlung der COPD, da Exazerbationen als Motor für das Fortschreiten der Erkrankung gelten, die Lebensqualität erheblich beeinträchtigen und häufig zu Krankenhauseinweisungen führen.

Der positive Effekt von Tiotropium auf das Exazerbationsrisiko wurde in den Studien UPLIFT und POET-COPD bestätigt. Dass das LAMA auch bei niedrigem Risiko (Gruppen A und B) die Exazerbationsrate reduziert und die Lungenfunktion verbessert, konnte jetzt in einer neuen Auswertung der UPLIFT-Daten demonstriert werden (Halpin D et al., ERS 2012, Wien, Poster P2190): die Hazard Ratio wurde für die Zeit bis zur ersten Exazerbation im Vergleich zur Kontrollgruppe signifikant verbessert $(0,76$ vs. $0,86, p<0,0001)$ und die durchschnittlichen jährlichen Exazerbationsraten signifikant vermindert $(0,43$ vs. $0,61, p<0,0001)$.

- Dagmar Jäger-Becker

Quelle: Pressekonferenz "Spiriva ${ }^{\circledR}$ - This is Your Life", ERS-Kongress, Wien, September 2012 (Veranstalter: Boehringer Ingelheim)

Ulcus cruris, Dekubitalulzera, diabetische Fußulzera

\section{Welche Wundauflage eignet sich für chronische Wunden am besten?}

- Eine Orientierungshilfe bieten Leitlinien wie die S3-Leitlinie "Lokaltherapie chronischer Wunden bei den Risiken CVI, PAVK und Diabetes mellitus" von der Deutschen Gesellschaft für Wundheilung und Wundbehandlung e.V. (DGfW). „Die lokale Wundtherapie soll eine ungestörte Wundheilung ermöglichen ...", zitierte Prof. Joachim Dissemond, Essen, und „... ein genereller Vorteil von Wundauflagen, die ein so genanntes "feuchtes Wundmilieu" ermöglichen, ist belegt."

Allerdings ist für die Zulassung von Wundverbänden kein Nachweis der klinischen Wirksamkeit und Sicherheit durch kontrollierte Studien notwendig. Daher stehen nur wenige Studien zur Verfügung, die eine evidenzbasierte Handlungsentscheidung erlauben. Eine Überlegenheit einzelner Wundprodukte konnte bislang nicht ausreichend belegt werden. Auch die S3-Leitlinie kommt zu dem Schluss, dass

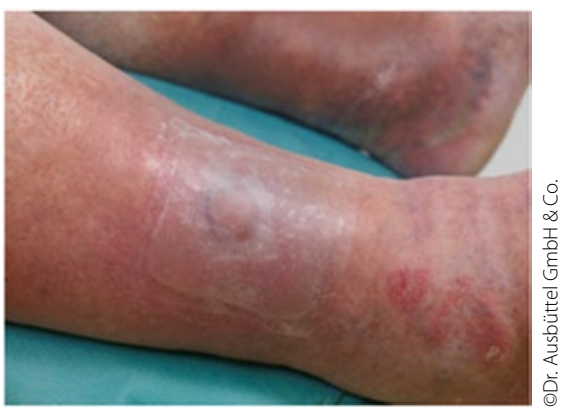

Da DracoHydro ultra transparent ist, kann die Wunde trotz Auflage beobachtet werden.

sich die Auswahl der Materialien insbesondere an Patientenkomfort, Exsudatmanagement, Kosten und individuellen Erfahrungen orientieren sollte.

Eine hydrokolloide Wundauflage, die diesen Anforderungen entspricht und sich durch ein günstiges Preis-Leistungs-Verhältnis auszeichnet, ist z. B. DracoHydro
Ultra (mit Hydrogeltechnologie). Sie kann bis zu sieben Tagen getragen werden, ist für einen phasenübergreifenden Einsatz geeignet, insbesondere bei mittelstark exsudierenden Wunden, ohne dass die Wunde verklebt und auch bei trockenen Wunden, da sie diese langanhaltend befeuchtet und so ein idealfeuchtes und wundheilungsförderndes Wundmilieu herstellt.

Eine weitere Wundauflage, die durch ihre Wirtschaftlichkeit überzeugt, ist die antimikrobiell wirksame Schaumstoffwundauflage DracoFoam PHMB. Die Alternative zu Silberwundauflagen kann problemlos bei EEG-, EKG-, Röntgen-, Ultraschall- und MRT-Untersuchungen getragen werden.

- Dr. Katrin Wolf

Quelle: Pressekonferenz „Sommergespräch: Moderne Wundversorgung - quo vadis?", Kassel, Juni 2012 (Veranstalter: Dr. Ausbüttel \& Co. $\mathrm{GmbH}$ ) 